\title{
Stable efficiency roll-off in phosphorescent organic light-emitting diodes
}

\author{
Sung Hyun Kim and Jyongsik Jang \\ School of Chemical and Biological Engineering, Seoul National University, Shinlim-dong, Kwanak-gu, \\ Seoul 151-742, Korea \\ Kyoung Soo Yook and Jun Yeob Lee ${ }^{\text {a) }}$ \\ Department of Polymer Science and Engineering, Dankook University, Jukjeon-dong, Suji-gu, Yongin, \\ Kyeonggi 448-701, Korea
}

(Received 4 November 2007; accepted 29 December 2007; published online 18 January 2008)

Origin of efficiency roll-off in phosphorescent organic light-emitting diodes was investigated with triplet mixed host devices and stable devices with little efficiency roll-off was developed. Efficiency roll-off was significant in the device with narrow recombination zone (RZ) and charge leakage out of emitting layer at high luminance was critical to efficiency roll-off. Efficiency roll-off could be reduced in triplet mixed host device with broad RZ and little charge leakage at high driving voltage. Triplet mixed host devices with an exciton blocking layer showed a quantum efficiency over $90 \%$ of maximum quantum efficiency at a luminance of $20000 \mathrm{~cd} / \mathrm{m}^{2}$. (c) 2008 American Institute of Physics. [DOI: 10.1063/1.2836270]

Phosphorescent organic light-emitting diodes (PHOLEDs) have a merit of high quantum efficiency and $100 \%$ theoretical maximum quantum efficiency was already reported. ${ }^{1}$ However, quantum efficiency of PHOLEDs tends to decrease at high current density because of triplet-triplet exciton quenching mainly originated from long triplet excited lifetime of phosphorescent emitting materials. ${ }^{2,3}$

There have been many studies on the origin of efficiency roll-off in PHOLEDs. Triplet-triplet annihilation at high current density was the main reason for the efficiency roll-off and it was proportional to the square of triplet exciton density. ${ }^{4}$ Triplet-polaron quenching was reported to be responsible for triplet efficiency roll-off through triplet energy transfer to charged molecules. ${ }^{3}$ The triplet-polaron quenching scales with triplet exciton density and its effect was not so significant as triplet-triplet quenching. Baldo et al. proposed that triplet-triplet annihilation is the main mechanism for efficiency decrease at high luminance. ${ }^{3}$ In addition to triplet-triplet annihilation and triplet-polaron quenching, dissociation of excitons into free charge carriers contributes to efficiency decrease at high current density. ${ }^{6}$ These triplet quenching processes mentioned above can be correlated with device structures and triplet devices with long triplet excited state lifetime showed serious triplet-triplet exciton quenching and narrow emission zone was found to have negative effect on triplet-triplet quenching because triplet exciton density is high in the exciton formation zone. ${ }^{7-9}$

Even though there have been some experimental studies about efficiency roll-off of PHOLEDs, there has been no systematic study about the relationship between efficiency roll-off and device structures. In this work, triplet efficiency roll-off was studied using triplet mixed host devices and the origin of the triplet efficiency decrease at high luminance was revealed by monitoring recombination zone (RZ) of triplet mixed host devices according to driving voltage.

Triplet mixed host device structures used in this work were indium tin oxide $(150 \mathrm{~nm}) / N, N^{\prime}$-diphenyl-

\footnotetext{
a) Author to whom correspondence should be addressed. Tel./FAX: 82-31-
} 8005-3585. Electronic mail: leej17@dankook.ac.kr.
$N, N^{\prime}$-bis-[4-(phenyl- $m$-tolyl-amino)-phenyl]-biphenyl-4, $4^{\prime}$ diamine $(60 \mathrm{~nm}) / N, N^{\prime}$-di(1-naphthyl)- $N, N^{\prime}$-diphenylbenzidine $[(\mathrm{NPB}) 30 \mathrm{~nm}] /$ light emitting layer $[(\mathrm{EML}), 30 \mathrm{~nm}] / 2$, 9-dimethyl-4, 7-diphenyl-1, 10-phenanthroline [(BCP) $5 \mathrm{~nm}] /$ tris $(8$-hydroxyquinoline $)$ aluminium $(20 \mathrm{~nm}) / \mathrm{LiF}$ $(1 \mathrm{~nm}) / \mathrm{Al} \quad(200 \mathrm{~nm})$. $4,4^{\prime}, 4^{\prime \prime}$,-tris $(N$-carbazolyl)triphenylamine (TCTA) and 1,3,5-tris ( $N$-phenylbenzimidazole-2-yl)benzene (TPBI) were host materials in EML and tris(2-phenylpyridine) iridium $\left[\operatorname{Ir}(\mathrm{ppy})_{3}\right]$ was a triplet dopant. TPBI contents in mixed host device were $0 \%, 25 \%, 50 \%, 75 \%$, and $100 \%$. In addition to standard mixed host devices, triplet mixed host devices with TCTA as an exciton blocking layer (EBL) between NPB and EML were also fabricated to study the origin of triplet efficiency roll-off of $\operatorname{Ir}(\mathrm{ppy})_{3}$ devices. To study the RZ of PHOLEDs, red emitting iridium(III) bis(2-phenylquinoline) acetylacetonate $\left[\operatorname{Ir}(\mathrm{pq})_{2}\right.$ acac $]$ was inserted as a sensing layer inside EML in three regions at a thickness of $0.5 \mathrm{~nm}$. The distances of red sensing layer from hole transport layer (HTL) were 5,

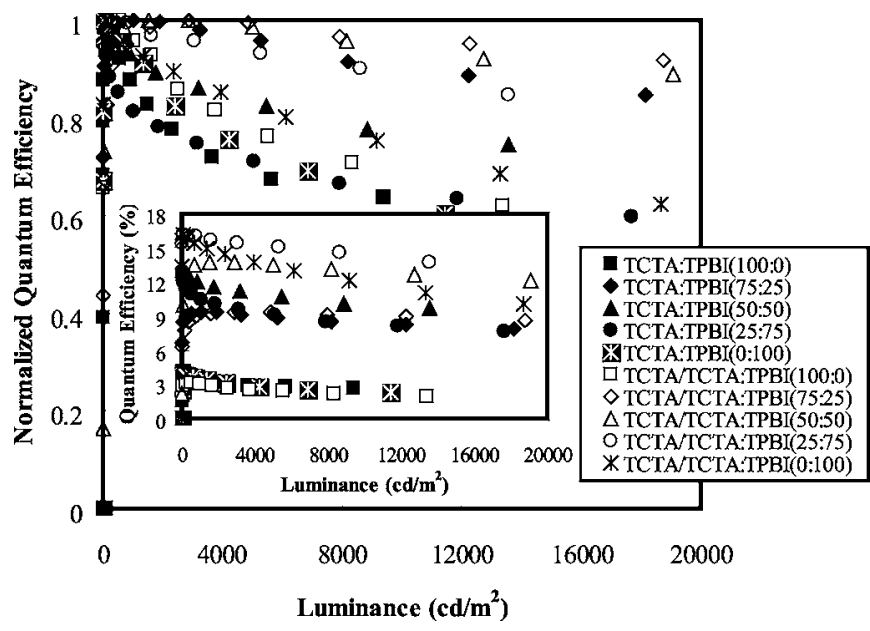

FIG. 1. Normalized quantum efficiency of TCTA:TPBI mixed host devices with and without TCTA exciton blocking layer (inset: quantum efficiency of TCTA:TPBI mixed host devices with and without TCTA exciton blocking layer). 


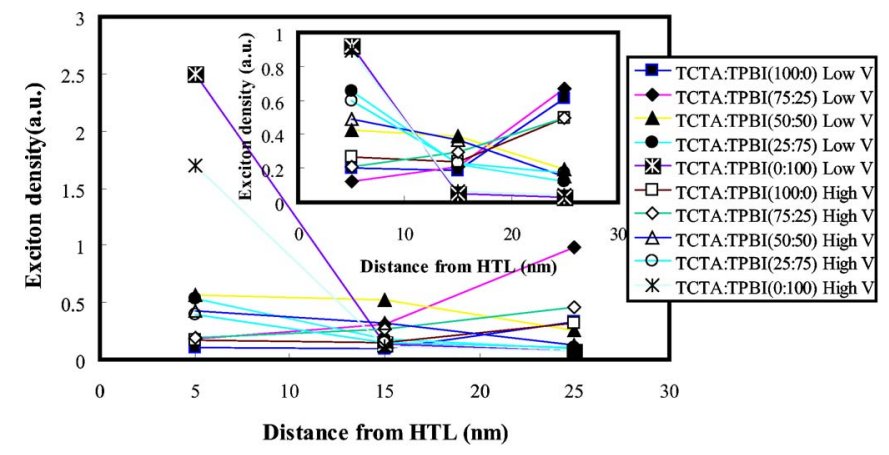

(a)

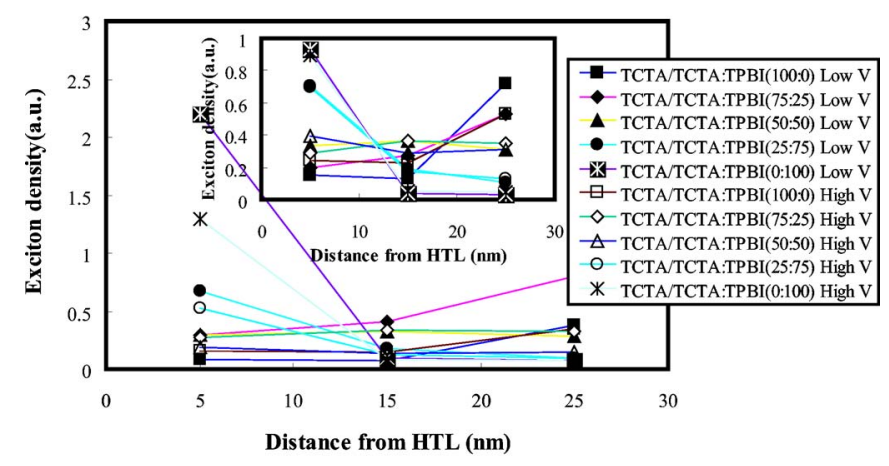

(b)

FIG. 2. (Color online) Exciton density of TCTA:TPBI mixed host devices according to distance from hole transport layer (inset: relatively normalized exciton density of TCTA:TPBI devices). (a) TCTA:TPBI devices without TCTA exciton blocking layer. (b) TCTA:TPBI devices with TCTA exciton blocking layer.
15, and $25 \mathrm{~nm}$. Current density-voltage-luminance characteristics and electroluminescence spectra of PHOLEDs were measured with Keithley 2400 source measurement unit and CS 1000 spectrophotometer.

It has been known that triplet lifetime of emitting materials and width of RZ are important for the efficiency roll-off in PHOLEDs. ${ }^{3,8}$ However, hole or electron leakage at high luminance and RZ change at high driving voltage are also important for the efficiency decrease at high luminance because there is a change of exciton density at different voltages. Therefore, investigation of RZ and charge leakage according to driving voltage can reveal the origin of efficiency roll-off in triplet devices.

Figure 1 shows quantum efficiency $(\mathrm{QE})$ and normalized QE of triplet mixed host devices with and without TCTA EBL. In general, TCTA:TPBI mixed host devices showed better performances than TCTA or TPBI standard devices and the QE was even better in TCTA:TPBI devices with TCTA EBL than TCTA:TPBI mixed host devices without EBL. High QE in TCTA:TPBI mixed host devices is mostly due to holes and electron balance in light emitting layer and additional improvement of QE by TCTA originated from blocking of triplet exciton quenching and further charge balance in EML. ${ }^{10}$

To compare efficiency roll-off in mixed host devices, QE of all devices was normalized and normalized QE is also shown in Fig. 1. TCTA EBL reduced the triplet efficiency roll-off in all devices and mixed host devices showed reduced efficiency roll-off compared with standard TCTA and TPBI devices. In terms of efficiency roll-off, TCTA:TPBI

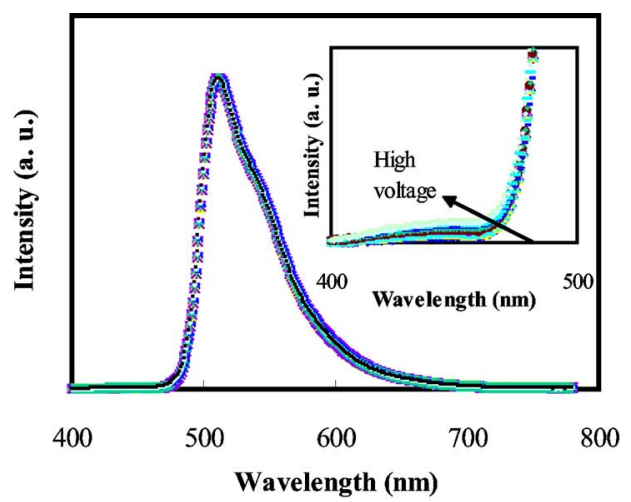

(b)

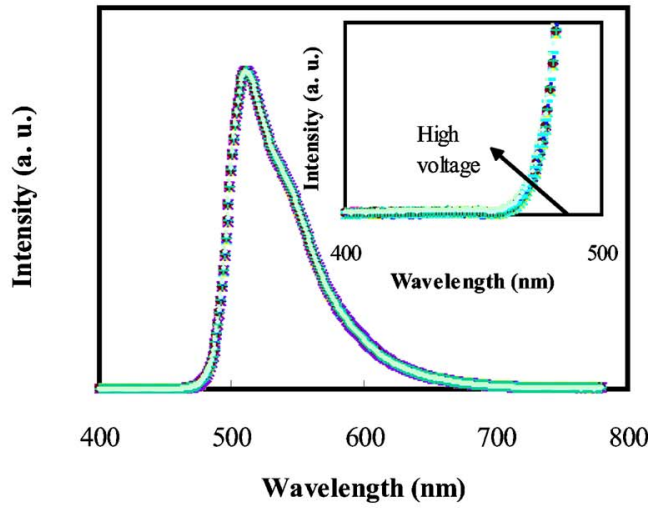

(d)

FIG. 3. (Color online) Electroluminescence spectra of TCTA:TPBI devices according to driving voltage. (a) TCTA:TPBI (50:50) device without exciton blocking layer. (b) TCTA:TPBI (75:25) device without exciton blocking layer. (c) TCTA:TPBI (50:50) device with exciton blocking layer. (d) TCTA:TPBI (75:25) device with exciton blocking layer.

Downloaded 19 Jun 2008 to 147.46 .244 .160 . Redistribution subject to AIP license or copyright; see http://apl.aip.org/apl/copyright.jsp 
(75:25) and TCTA:TPBI (50:50) devices with TCTA EBL exhibited the best performances and the normalized QE was over $90 \%$ of maximum $\mathrm{QE}$ at $20000 \mathrm{~cd} / \mathrm{m}^{2}$. This value is better than any other efficiency roll-off value reported in triplet devices and it was possible to get stable efficiency value from 1000 to $20000 \mathrm{~cd} / \mathrm{m}^{2}$ in these devices.

To study the relationship between efficiency roll-off and light emission behavior of PHOLEDs, RZ of all devices was measured using red sensing layer. Assuming that relative red intensity represents exciton density in EML, exciton density can be calculated by integrating red emission peaks. Figure 2 summarizes calculated exciton density in three regions of EML and relatively normalized exciton density is shown as an inset. Exciton density was plotted against the distance of red sensing layer from HTL. TCTA devices showed high exciton density near BCP, while TPBI devices showed high exciton density near HTL. In case of mixed host devices, rather broad emission zone was observed and emission zone was shifted from BCP side to HTL side at high driving voltage. Comparing exciton density of mixed host devices without EBL at low voltage and high voltage, TCTA:TPBI (75:25) and TCTA:TPBI (50:50) showed more uniform exciton distribution in EML than other devices. In particular, TCTA:TPBI (75:25) device showed broader emission zone at high voltage than at low voltage, indicating that triplet-triplet exciton quenching can be reduced at high voltage. However, TCTA:TPBI (50:50) devices showed rather narrow emission zone at high voltage, which had negative effect on triplettriplet exciton quenching. In addition, strong NPB emission at high voltage (Fig. 3) is also responsible for increased efficiency roll-off in TCTA:TPBI (50:50) devices compared with TCTA:TPBI $(75: 25)$ devices. Therefore, TCTA:TPBI (75:25) devices showed the best efficiency roll-off behavior among all mixed host devices without EBL.

Similar trend was observed in mixed host devices with TCTA EBL. TCTA:TPBI (75:25) and TCTA:TPBI (50:50) devices showed uniform exciton density over whole EML [Fig. 2(b)]. RZ of TCTA:TPBI (75:25) was rather narrow at low voltage, but it became uniform at high voltage, contributing reduced exciton quenching at high voltage. Even though TCTA:TPBI (50:50) also showed uniform distribution of exciton at low and high voltages, the RZ became relatively narrower at high voltage, leading to rather high efficiency roll-off compared with TCTA:TPBI (75:25) device, as shown in Fig. 1. Considering that there was little NPB emission in all mixed host devices with EBL over all voltage range (Fig. 3), narrow RZ of TCTA:TPBI (50:50) device at high voltage is responsible for rather high efficiency roll-off in TCTA:TPBI (50:50). In mixed host devices, TCTA:TPBI devices with EBL showed more uniform exciton density than TCTA:TPBI devices (Fig. 2) and reduced NPB emission (Fig. 3), which is responsible for reduced efficiency roll-off in TCTA:TPBI mixed host devices with TCTA EBL.

In summary, distribution of RZ and charge leakage out of EML at high voltage were critical to efficiency roll-off in PHOLEDs. Broad RZ had positive effect on reducing efficiency roll-off because of low probability of triplet-triplet exciton quenching. In particular, RZ broadening at high voltage was effective to reduce efficiency roll-off in triplet devices. In addition, complete blocking of charge leakage at high luminance was important to get improved efficiency roll-off in triplet devices and TCTA EBL was efficient to prevent charge leakage out of EML.

This work was supported by Grant No. RTI04-01-02 from the Regional Technology Innovation Program of the Ministry of Commerce, Industry and Energy (MOCIE).

${ }^{1}$ C. Adachi, M. Baldo, M. E. Thompson, and S. R. Forrest, J. Appl. Phys. 90, 5048 (2001).

${ }^{2}$ M. A. Baldo, M. E. Thompson, and S. R. Forrest, Pure Appl. Chem. 71, 2095 (1999).

${ }^{3}$ M. A. Baldo, C. Adachi, and S. R. Forrest, Phys. Rev. B 62, 10967 (2000).

${ }^{4}$ R. G. Kepler, J. C. Caris, P. Avakian, and E. Abramson, Phys. Rev. Lett. 10, 400 (1963).

${ }^{5}$ V. Ern, H. Bouchriha, J. Fourny, and G. Delacote, Solid State Commun. 9, 1201 (1971).

${ }^{6}$ J. Kalinowski, W. Stampor, J. Mezyk, M. Cocchi, D. Virgili, V. Fattori, and P. Di Marco, Phys. Rev. B 66, 235321 (2002).

${ }^{7}$ M. A. Baldo and S. R. Forrest, Phys. Rev. B 62, 10958 (2000).

${ }^{8}$ S. Reineke, K. Walzer, and K. Leo, Phys. Rev. B 75, 125328 (2007).

${ }^{9}$ S. Reineke, G. Schwartz, K. Walzer, and K. Leo, Appl. Phys. Lett. 91, 123508 (2007).

${ }^{10}$ S. H. Kim, J. Jang, and J. Y. Lee, Appl. Phys. Lett. 90, 223505 (2007). 